\title{
Necrotic Control of the Aesthetics of Evolved Art
}

\author{
Daniel Ashlock and Julie Greensmith
}

\begin{abstract}
This study uses necrosis, a technique from the domain of artificial immune systems, to control the evolution of apoptotic cellular automata. These automata generate complex images that require a very small amount of initial data. The genes that yield these images are embedded in an extremely complex adaptive landscape. The process of controlling the type of images located by applying necrosis is found to be a simple and efficient technique, in comparison to writing more complex fitness functions for the original evolutionary computation system. Two kinds of necrosis are tested, a soft shape based system and a crisp entropy based system. Both sorts of necrosis are found to be able to steer evolution effectively, with the shape based necrosis working well, and the entropy based necrosis having some problems when more extreme forms of necrosis driven filtration are employed. Possible generalizations to steering other evolutionary optimization tasks are outlined.
\end{abstract}

\section{INTRODUCTION}

With the advent of Artificial Immune Systems [15] within the domain of computational intelligence, a number of techniques inspired by natural immune systems have been added to the techniques available for nature inspired computation. In this study we add necrosis to an evolutionary algorithm that located apoptotic cellular automata [3]. Necrosis is the violent disruption of a cell caused by some form of stress, leading to its recognition by the immune system as being associated with foreign material as part of the detection of non-self entities. In this process the cell itself gives off biochemical signals that the cell itself represent danger or damage to tissue cells e.g. heat shock proteins, uric acid. In this study, necrosis is added to an existing evolutionary algorithm as a secondary form of selection operator that zeros out the fitness of population members with undesirable properties. This is presented as an alternative to trying to write complex fitness function that bake constraints into a single feature of the algorithm as was done in [4].

Cellular automata are a type of discrete model of computation. A cellular automata has three parts,

1) A collection of cells divided into neighborhoods of each cell. In this study this is a linear array of cells with neighborhoods consisting of a cell and its four nearest neighbors.

2) A set of states that cells can have. In this study we use the numbers $0-7$ as states.

3) A rule that maps the set of possible cell states of a neighborhood to a new state for the neighborhood. This rule is described subsequently.

Daniel Ashlock is with the Department of Mathematics and Statistics at the University of Guelph, in Guelph, Canada email: dashlock@uoguelph.ca

Julie Greensmith is with the School of Computer Science at the University of Nottingham, UK, email: Julie.Greensmith@nottingham.ac.uk

The authors thank the Universities of Guelph and Nottingham for their support of this work
Cellular automata (CA) can be described as discrete dynamical systems that exhibit self-organizing behaviour. A cell population evolves according to local transitions rules. The updating according to the transition rules may be synchronous or asynchronous. This study uses synchronous updating. CA can be used as models for complex natural systems that contain large numbers of identical components experiencing local interactions [29], [22].

CA have been applied to the study of a diverse range of topics, such as structure formation[7], heat conduction[8], language recognition[20], traffic dynamics[17] and cryptography[2], to name a few. CA have also been used for more aesthetic purposes, such as image and sound generation. Serquera and Miranda of the Interdisciplinary Centre for Computer Music Research, UK, have published many works on the use of CA for sound synthesis [24], [1]. Much of their work consists of mapping the histogram sequence of a CA evolution onto a sound spectrogram, which produces spectral structures evolving in time. It is claimed that the mapping produces a "natural" behaviour, and can replicate acoustic instruments[25].

CA have also been applied to the visual arts. CA have been used to produce artistic images[5], [19], [4], [13], in the form of time histories of one-dimensional cellular automata with states shown as colors. The use of CA has been extended to the fields of architecture and urban design[26], [11]. An interesting application has been the use of CA in simulating the emergence of the complex architectural features found in ancient Indonesian structures, such as the Borobudur Temple [27]. Ashlock and Tsang[5] produced evolved art using 1dimensional $\mathrm{CA}$ rules. $\mathrm{CA}$ rules were evolved using a string representation. The $\mathrm{CA}$ either underwent slow persistent growth, or planned senescence. The resulting fitness landscapes were rugged with many local optima. This led to the production of aesthetically pleasing images. This study revisits these fitness functions with a more general space of cellular automata rules.

In this study we return to earlier work that demonstrated that the fitness landscape of even the relatively simple encoding of CA used in this study has a complex fitness landscape [3]. In [4] the algorithm for finding CA rules was modified to restrict the shape of the rendered time-histories. Here we employ necrosis to eliminate undesirable CA in two different ways. The necrosis is applied to the rendered images derived from the CA rules, meaning that the selection criteria related to necrosis can be specified in a manner directly related to the desired appearance. Two different selection criteria are used, the first uses a desired shape for the image while the second places bounds on the entropy of state usage, both 
upper and lower.

\section{A. Incorporating the Danger Theory}

The Danger Theory emerged as a complementary idea to the classical self-nonself dogma of immunology, postulating that the human immune system requires the detection of damage to the body in order to mount an effector response[18]. Danger Theory states that the detection of nonself matter i.e. antigen, is insufficient to activate the cells of the immune system. Full immune activation can only occur when both nonself antigen and danger are detected. Danger signals are detected by the antigen presenting cells of the immune system, including a class of cells termed dendritic cells

Danger signals are released into the body as a consequence of uncontrolled cell death as a result of a process termed necrosis. Necrosis is caused by one of four triggers: extreme heat, extreme cold, lack of oxygen and infection by bacteria or viruses. Despite both being forms of cell death, apoptosis is the antithesis of necrosis, and can be described as controlled cell death. The human immune system has the sensing capability to discriminate between apoptosis and necrosis through somatically encoded receptors on an antigen presenting cell.

Necrosis causes activation of T-helper cells through the maturation and migration of dendritic cells, ultimately leading to the elimination of the entity responsible for causing the necrosis in the first instance. Apoptosis causes tolerance in T-helper cells via suppressing the maturation process of dendritic cells which leads to tolerance of matter present during apoptotic processes. Monitoring the balance between apoptotic and necrotic processes in tissue cells is a fundamental process in immunology. In this study we use apoptosis to regulate the growth of the cellular automata while necrosis controls which type(s) or automata with controlled growth, are permitted to survive.

The Danger Theory was first proposed by Matzinger in 1994 [18], though it took nearly a further 20 years to amass sufficient evidence to ground the theory empirically [21]. The precise molecular signalling pathways and corresponding receptors have been elucidated, adding weight to the validity of the theory despite widespread initial criticism of the Danger Theory. The essential role of antigen presenting cells is highlighted as the key cell type responsible for the collection and analysis of Danger Signals and the complementary 'safe' signals of apoptosis.

Artificial Immune Systems (AIS) are computational techniques inspired by the function and behaviour of the human immune system [15]. Necrosis is a feature of AIS which encompass the Danger Theory, first proposed by Aickelin and Cayzer (2002)[9]. The use of both apoptosis and necrosis features heavily in the research towards the development of the Dendritic Cell Algorithm [14], which uses the balance between the 'safe' signals of apoptosis and the 'danger' signals of necrosis as the basis of classification of anomalies[12]. The majority of Danger Theory inspired approaches use some version of the DCA to interrogate signals of necrosis or apoptosis and to perform some form of binary classification, as reviewed in Chelly and Elouedi (2016)[10].

In AIS literature there are many different interpretations of the Danger Theory, with applications ranging from management of wireless sensor networks through to the security of computer networks[23], or the approach incorporating algorithms which control the release of danger signals from metaphors of tissue as in [28] and [6]. At the core of most danger-based AIS is the detection of some form of danger signal, mapped to a set of underlying feature vector or stream data. The related technique, the DCA, uses a categorisation of underlying data streams into the necrotic 'danger stream' or the apoptotic 'safe stream'[16], where a multi-agent algorithm performs distributed signal processing. This algorithm centres around the analysis of the behaviour of the cell agents to reveal anomaly scores. In this research we aim to use a 'pure' Danger Theory approach i.e. focus purely on the processes of apoptosis and necrosis, in a manner similar to that used by Bentley et al. (2005)[6], without employing a population of artificial dendritic cells. Previous research has included an apoptosis operator into cellular automata. Given the similarity to the use of the concept of apoptosis in dangerbased AIS we aim to determine how a necrosis operator can also be integrated.

We explicitly declare two distinct operators for apoptosis and necrosis, which have distinct effects on a cellular automata. In terms of the Danger Theory, each automata in analogous to an individual cell in biological tissue, which has the capability to undergo either apoptosis or necrosis. The former process eliminates cells that undergo uncontrolled growth while the latter process eliminating members of the population that exhibit other undesirable properties. This is a novel approach to using the Danger Theory metaphor, focusing on the tissue cell aspects instead of models of the dendritic cell's processes. This also creates a terminological conflict as individual cellular automata have "cells" but the entire automata is treated as a cell in the metaphor. The usage should be clear from context.

Upon meeting a set of criteria we can introduce either type of cell death to individuals in the automata population. Death by necrosis limits the automata's growth based on a metaphor of introducing unexpected 'necrosis' to individuals, rendering areas of the landscape unusable. This approach explicitly encapsulates the metaphor of using distinct necrotic and apoptotic processes incorporated into the growth of an evolvable cellular automata, as detailed in the methodology section of this paper. This study aims to show that the addition of a necrosis operator produces significantly different automata than whose produce only with the apoptosis operator introduced previously.

The rest of this study is structured as follows. In Section II we give the design of experiments, including the representation, fitness functions, and analysis tools. In Section III the results are presented and discussed. Section IV we draw conclusions and discuss potential next steps. 


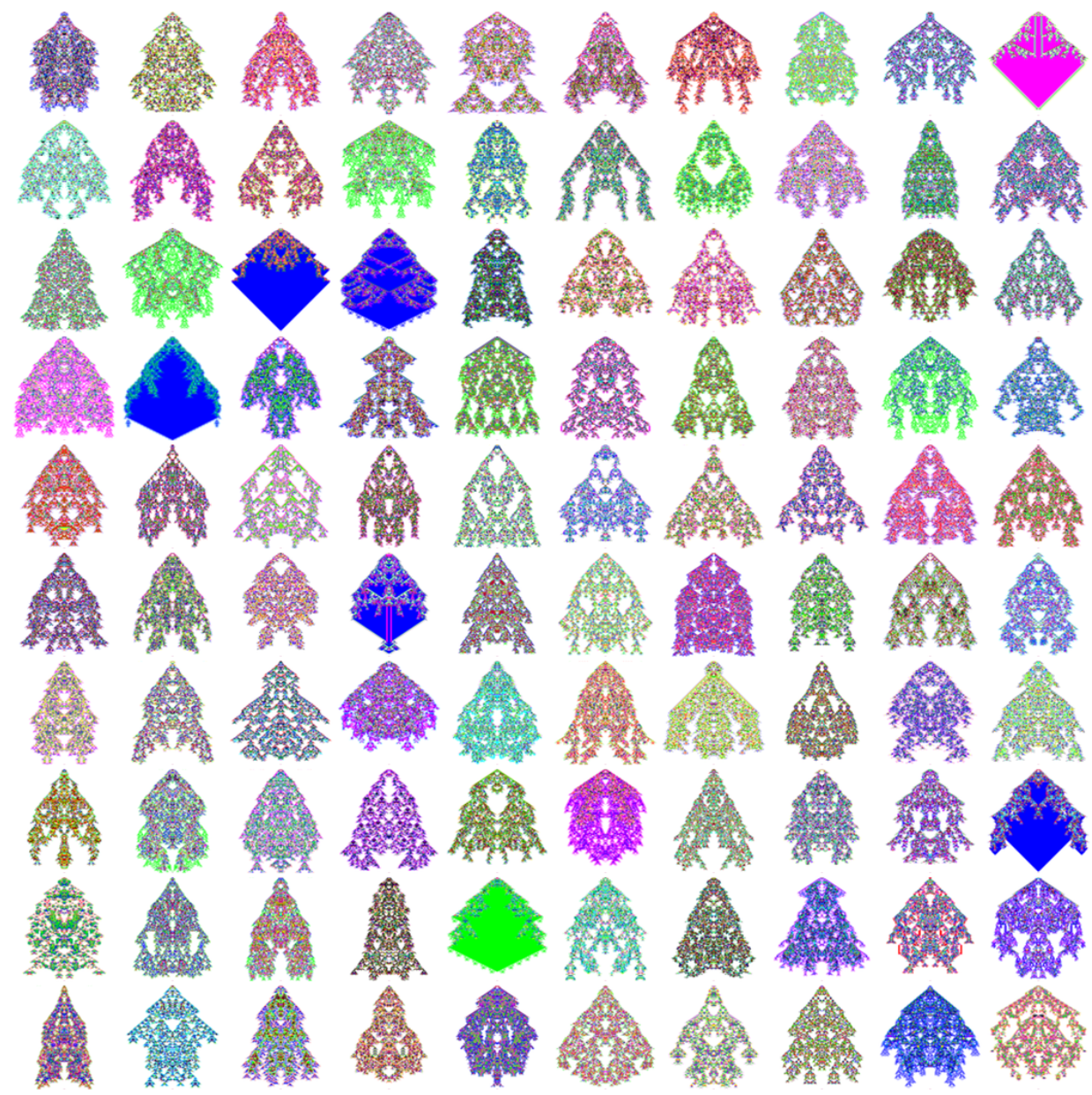

Fig. 1. Shown are renderings of time histories of 100 evolved apoptotic cellular automata.

\section{DESIGN OF EXPERIMENTS}

Four sets of experiments are performed. The first performs a parameter study on the mutation rate for locating apoptotic cellular automata not constrained by necrosis. This parameter study had not been performed in earlier studies and locates a best value for the mutation rate not used in the earlier work. The second set of experiments uses four shape restrictions and a soft necrosis selection step to steer the automata rules into favouring particular parts of the search space. The third set of experiments used a crisp form of necrosis based on the Shannon entropy of state usage. This type of necrosis affects the state usage of the automata, indirectly controlling shape, and steering the automata in a manner that favors different portions of the fitness landscape. The fourth set of experiments verifies that one of the shapes used in the second set of experiments scales properly. The details of these experiments and the evolutionary algorithm is given in the remainder of this section.

The cell states for the automata used in this study are the numbers $\{0,1,2,3,4,5,6,7\}$. The cell sets of the automata are one-dimensional arrays of 201 in the first three sets of experiment and 601 cells in the last set. Automata that reach the edges of the cell array are awarded zero fitness, preventing dependence of the auotmata's rendering on the width of the drawing arena. The neighborhood of each cell consists of the cell itself and two cells to the left and right of it. The rules for the cellular automata are the target of evolution. They are arrays specifying 36 choices of cell states. Rules are applied as follows - the numbers in the five cells are summed, yielding a number in the range 035. This number is used as an index to look up the new state of the cell in the center of the neighborhood in the array. State zero is designated at the quiescent state of the automata and the first entry of the rule, corresponding to the neighborhood [00000], is forced to be zero so that a completely quiescent neighborhood yields a quiescent cell. This permits a natural definition of living (non-quiescent) and dead (quiescent) cells.

The representation used for evolution is a string representation, with the string data structure being the array giving the values of the updating rules. The variation operators used are two point crossover and $k$-point mutation in which $k$ values within the updating rule are changed. Given that the first cell of the array is forced to be zero, there are $8^{35} \cong 1.14 e+68$ rules in the search space. Tens of thousands of different local optima of the search space have been identified in the 
research thus far. The complexity of the fitness landscape for this space is documented in [3]. The fitness of the cellular automata in this study are computed using the time history of the automata. Examples of such histories appear in Figure 1. All the automata in this study use the apoptotic fitness function, named after the biological process of programmed cell death.

Definition 1: Apoptotic fitness is zero if the time history contains any living cells when the time history reaches the bottom of the rendering array. Otherwise the fitness is the number of live cells in the time history from time $t=0$ until the automata ceases to produce live cells.

Apoptosis in these automata is triggered by hitting the bottom of the rendering array. This is separate from necrosis which is triggered by recognition of other undesirable qualities in the rendering of the automata.
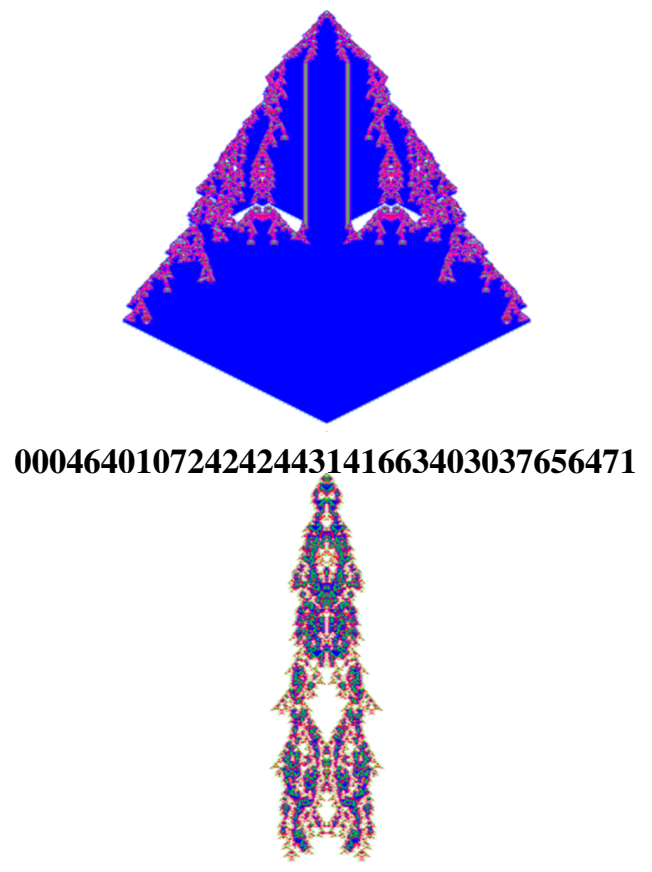

\section{1}

Fig. 2. Shown are renderings of time histories of an automata grown without necrosis (top) and one with necrosis triggered by leaving the middle quarter of the rendering area (bottom). The evolved rules for the automata are also shown. These automata were produced with an evaluation size of $601 \times 601$ in the fourth group of experiments.

\section{A. Evolutionary Algorithm Design}

The evolutionary algorithm in this study, before adding necrosis, is a standard one. The cellular automata updating rules are stored as strings of 36 integers with values in the range $0-7$, corresponding to the cell states. Two variation operators are used: two point crossover of the string and $k$-point mutation that replaces the value at at $k$ positions selected uniformly at random within the rule. Selection and replacement are accomplished with generational size-four tournament selection. The population is shuffled into groups of four CA-rules. The two more fit are copied over the two less fit. The copies are subjected to crossover and mutation. Such an updating is called a generation. In each experiment, the algorithm is run for 6000 generations with a population of 200 automata rules. A collection of 100 replicates with different random number seeds is used in each experiment.

The algorithm is modified as follows to implement Necrosis. The entire population is passed, one at a time, to the necrosis test. If they fail this test, they are recognized as emitting danger signals their fitness is reduced to zero. Necrosis is applied immediately after reproduction and fitness evaluation of new structures. Both old and new structures are tested for by the necrosis filter and the data needed to perform the necrosis test is recorded in the same manner that fitness is, in a global array. The necrosis functions used in the various experiments are given below.

Mutation rate experiments In these experiments the maximum number of mutations (MNM) is set to 1, 2, 3, 4 , and 5. The actual number of mutations performed is selected uniformly at random in the range one to MNM. These experiments do not use necrosis.

Soft necrosis shape experiments In these experiments a shape specification is used to designate positions within the rendered automata that cause the emission of danger signals. Four shapes are used:

- Outside of the middle half of the rendering arena, in the $x$-coordinate, the middle half restriction.

- Outside of the middle quarter of the rendering arena, in the $x$-coordinate, the middle quarter restriction.

- Outside of a square with corners in the center of each side of the rendering arena, the diamond restriction.

- Positions within the lower quadrant of the drawing arena, $y>x$ and $y>1-x$ intersected with the middle half of the square in the $x$ direction. The limited wedge restriction.

To apply necrosis, the number of living cells $n$ outside of the shape was totalled and a CA rule was found to be emitting danger signals by comparing a uniform random number in the range $[0,1]$ to the function given in Equation 1.

$$
p(n)=\frac{e^{\left(\frac{n}{K}-r\right)}}{e^{\left(\frac{n}{K}-r\right)}+1}
$$

The value $K$ scales the number of cells, $n$, that are out of bounds and $r$ is an offset. Function 1 is the probability of necrosis, conditioned on the number of cells outside of the shape. The values $K=1000$ and $K=2000$ are used with $r=2$ The parameter $K$ controls the sharpness of the sigmoid probability curve given by equation 1 while $r$ is an offset that lowers the chance of necrosis as $r$ increases.

Crisp necrosis entropy experiments The number of each type of living state appearing in a rendering are compiled an divided by the total number of living cells to create empirical probabilities $p_{i}$ of use for each living state $i$. The Shannon entropy of this distribution, given in Equation 


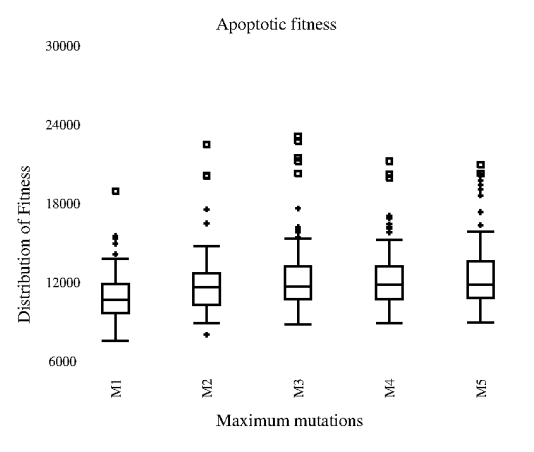

Fig. 3. Shown are the distribution of fitness values for the five experiments with different mutation rates for the baseline evolutionary algorithm for evolving apoptotic cellular automata, without necrosis.

2, is used to perform necrosis. Necrosis is not applied to low fitness (small) rendered automata to prevent necrosis from operating on automata with a small number of living cells. The smallness restriction on applying necrosis was implemented after many runs, with the lowest upper bound on acceptable entropy values, terminated with all individuals of zero fitness. An automata rule is found to be emitting danger signals if their entropy exceeds, or fails to exceed, a given bound. Three experiments encourage low entropy, three encourage high entropy. In the experiments in this study we set the smallness value to avoid necrosis to 200 living cells, i.e. necrosis is not applied to population members with a fitness below 201.

The entropy bounds used as the threshold for emitting danger signals are (greater than) 0.8, 1.0, and 1.2 and (less than) 1.0, 1.2, and 1.4. The maximum possible entropy for even use is $\log _{2}(7) \cong 2.81$. These entropy bounds were selected by preliminary experimentation, to avoid runs in which there are few or no automata rules whose with the desired level of entropy. The low entropy necrosis filters will encourage pictures that use few non-white colors or encourages very uneven usage of colors in the renderings. The high entropy filters encourage the relatively even use of many colors in the renderings.

$$
E=-\sum_{i=1}^{m} p_{i} \cdot \log _{2}\left(p_{i}\right)
$$

\section{RESUlTS AND DISCUSSION}

The mutation rate study on the algorithm without necrosis yielded the fitness distributions shown in Figure 3. The difference between $M N M 3$, 4, and 5 was not significant, but $M N M=3$ yielded the best upper outliers and so was chosen for the remaining simulations. This is in contrast with $M S M=1$ used in earlier studies. The example automata shown in Figure 1 are those found for the $M N M=3$ experiment without necrosis and should act as a basis for comparison with the automata found with the various necrosis filters active.

The fitness values for the shape-based and entropy-base experiments with necrosis active are shown in Figures 4

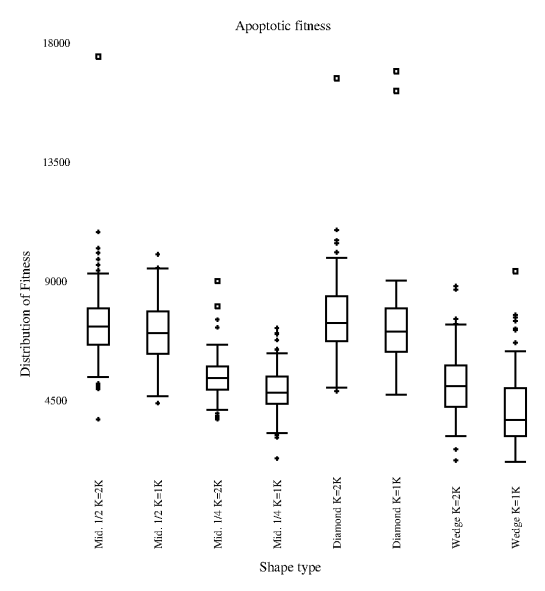

Fig. 4. Shown are the distribution of fitness values for the eight experiments performed with soft shape-based necrosis.

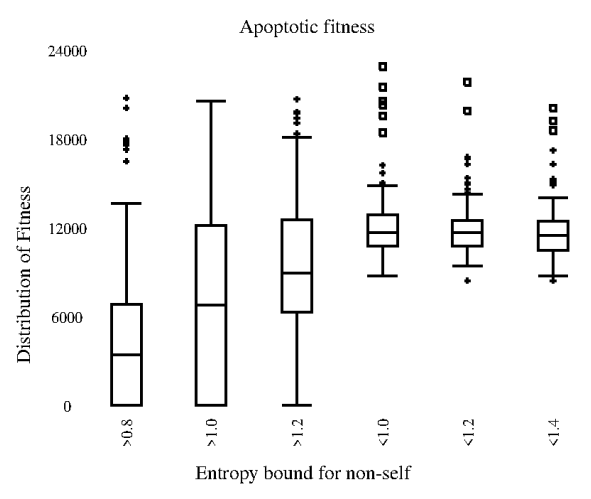

Fig. 5. Shown are the distribution of fitness values for the six experiments performed with crisp entropy-based necrosis.

and 5. The shape based experiments have lower fitness values than the experiments in the mutation rate study. The entropy results that recognize high entropy as a trigger for necrosis yield even lower fitness values. The experiments that recognize low entropy as a necrosis trigger have fitness distributions similar to the baseline experiments, and the automata located in these experiments are most similar to those in the baseline experiments.

The fitness data provide evidence that the algorithm is being diverted into different parts of the search space by the application of necrosis. Examination of the 1900 images produced located a single repeated image with a fitness over 200. The rules that produced this image were not identical, but the loci used in those rules during rendering are identical. This highlights a problem with documenting that necrosis is producing different results: repeated optima are rare because the number of optima is huge.

The low fitness of the experiments, those that recognize high entropy values as triggering danger signals, resulted in many runs that ended with cellular automata whose renderings were inside the safe size bound to avoid application of necrosis. Examples of such automata with small renderings are shown in Figure 6. These automata are two-thirds of all the runs with the experiment with necrosis trigger $E>0.8$. 


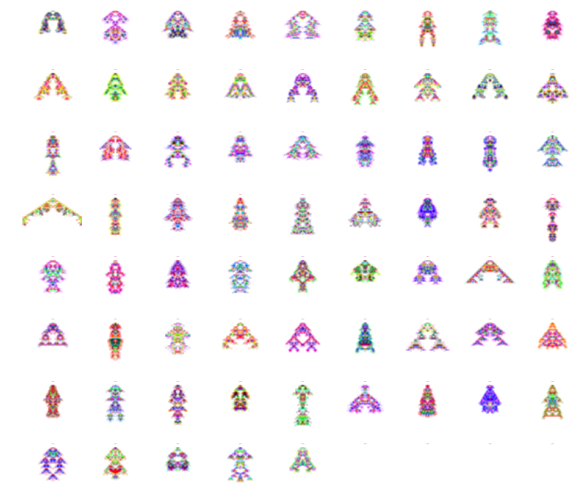

Fig. 6. Shown are renderings of low fitness automata that never managed to break out of the situation where the minimal size before application of necrosis preserved them. These are taken from the run that applied necrosis when the living state-use entropy exceeded 0.8

This result demonstrates the difficulty of locating highfitness, low entropy automata.

Evaluating the success, beyond the problem with many small pictures in some of the experiments encouraging low living-state entropy, requires examining the renderings of the evolved automata rules. Exemplary results from the experiments with soft necrosis based on shapes are shown in Figure 7. All four shapes used for necrosis are clearly visible in the example renderings. Two of the shapes, the last middle quarter and the third limited wedge have a small, but positive chance of being assigned zero fitness by the necrosis operator. This shows a feature of using soft necrotic selection - it tolerates minor violation of the shape restriction. If a cellular automata has only a small number of cells in the area that triggers necrosis, there is a good chance it will survive long enough to reproduce and may end up as the best organism in its run if it arises near the end of evolution.

Contrasting the renderings of rules located with different necrosis operators also demonstrates that evolution is being steered into different parts of the fitness landscape. All four shapes used in this study had a large impact on the images produced and, more to the point, they had the intended impact of causing the cellular automata to growing cells in the areas that triggered necrosis.

The results from the entropic experiments were not as strong as the shape based results. Exemplary results from the experiments with crisp necrosis based on state-use entropy are shown in Figure 8. In the results shown in Figure 8 the entropy, and hence diversity, of states used is biased up or down as intended. The failure is in the runs encouraging low entropy. The runs with the lowest acceptable upper bound on the entropy value failed two-thirds of the time, in the sense that the best-of-run results were those that never exceeded the fitness bound below which necrosis was not triggered. The renderings of the failed runs are shown in Figure 6.

The acceptable entropy values in the entropic experiments are nested - having entropy less than 0.8 , for example, automatically makes the entropy less than 1.2 . The nesting runs in opposite directions for the experiments encouraging high state-use entropy and low state-use entropy. The results in this study strongly suggest that renderings with high entropy of living states are more common than those with low entropy. This in reflected in the large numbers of failures to locate automata with large renderings when the entropic bound for danger signals was set to $E>0.8$.

The different restrictions on the use of colors in renderings implemented as entropy restrictions yield pictures with different appearances. The high entropy images have similar complexity of shape to the low entropy results, but greater chromatic complexity. The affects the aesthetics of the images, color palette and use are important artistic factors, but the impact of the entropy restrictions in this study was not particularly predicable. More intentional impact of necrosis filters on aesthetics is a topic for future work.

\section{Conclusions And Next StePs}

This study demonstrates that necrosis, based on danger theory, can be used to exclude portions of the fitness landscape in an evolutionary algorithm. Necrosis filters can steer the evolution of automata rules to ones with that avoid the properties negatively encoded by the definitions for emission of danger signals. This is a strong positive result for the technique, which permits us to decompose the imposition of restrictions on the search space from the fitness function. Such a decomposition simplifies algorithm design, in contrast with earlier work in which shape restrictions were incorporated into the fitness functions [4].

The entropic danger signals, when used to drive necrosis, are novel to this study. Placing very low upper bounds on the acceptable entropy values caused a two-thirds failure rate; higher upper bounds on entropy experienced much lower failure rates and placing a lower bound on acceptable entropy values for the distribution of cell states of living cells in the automata rendering did not cause failure. This tells us something not previously known about the distribution of entropy values within the fitness landscape - high entropy rules are more common.

An unexplored possibility is to stack the necrotic filters. This would be as simple as calling two distinct filters, one after the other. If, for example, we filtered for relatively high entropy and the middle quarter shape, we conjecture the results would be more even color use in automata that stuck near the center of the rendering arena. An under-explored area in this study is the use of soft or hard application of the necrosis rules. It might, for example, be the case that soft selection for low-entropy rules might alleviate the failure rate of low-entropy automata.

This study demonstrates that necrosis driven by danger signals in the form of detection of undesirable features are useful in the management of evolution across a challenging fitness landscape. This technique could be applied in many other areas of evolutionary computation. The technique could be respecialized, by recognizing known optima as dangerous, to permit the enumeration of optima. It might also be useful for encouraging exploration of the Pareto-frontier in multi-criteria evolutionary optimization. Exploration of other 

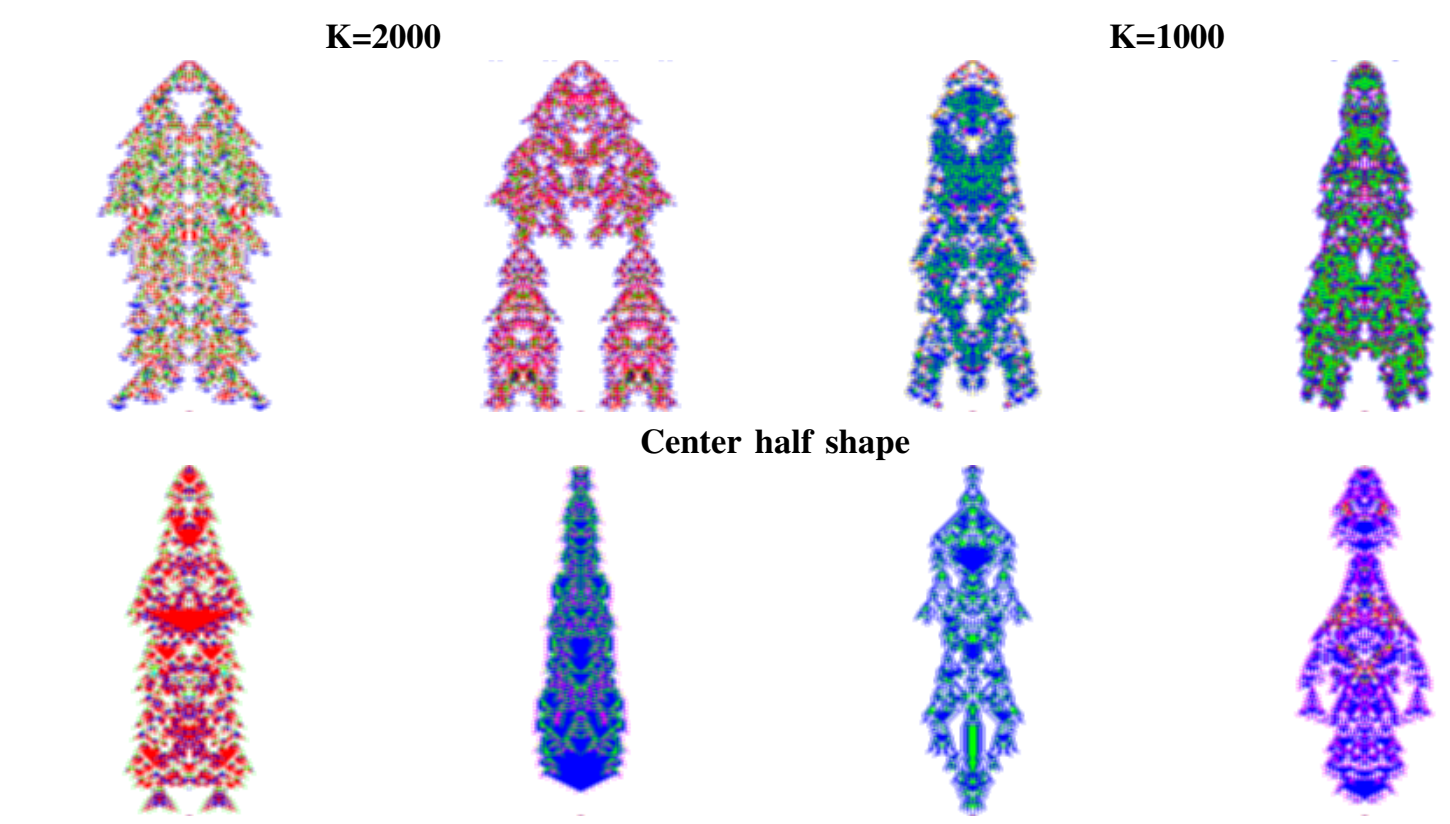

\section{Center half shape}
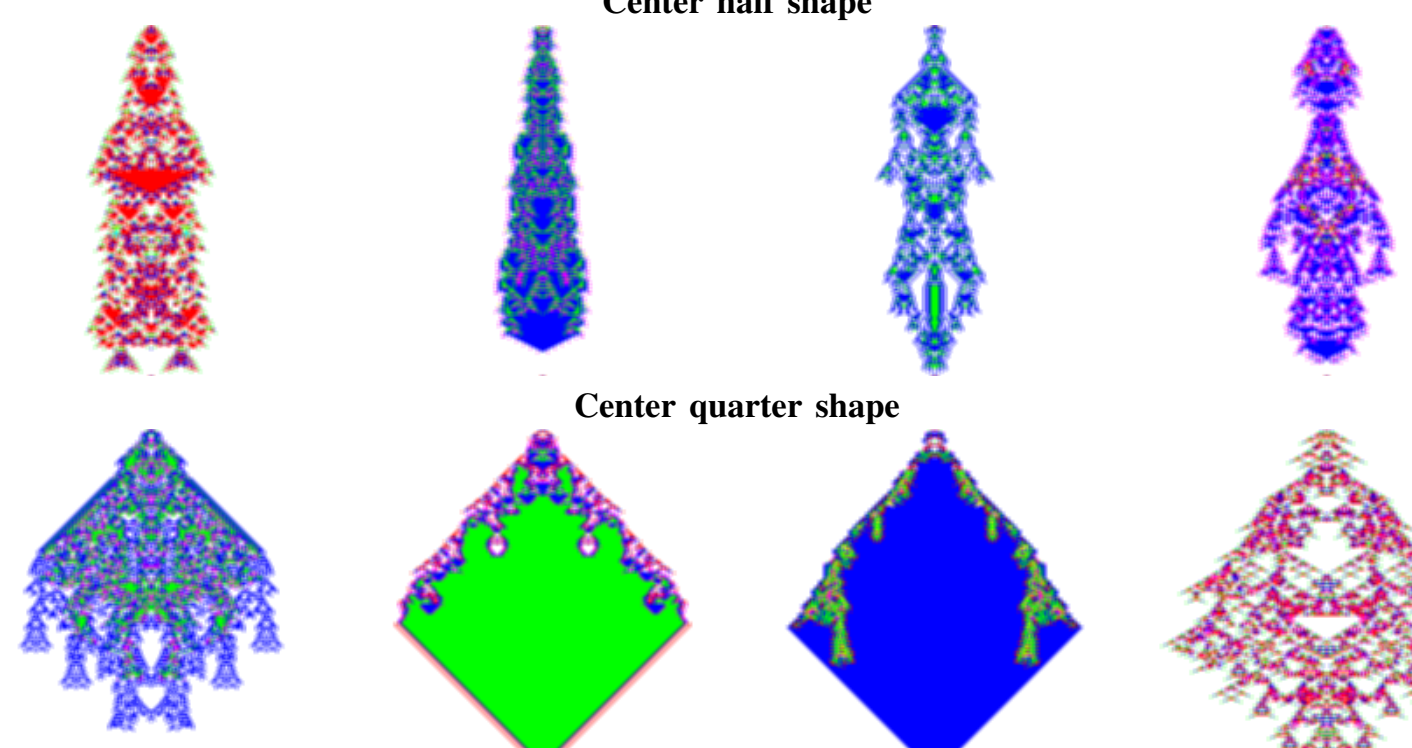

Center quarter shape
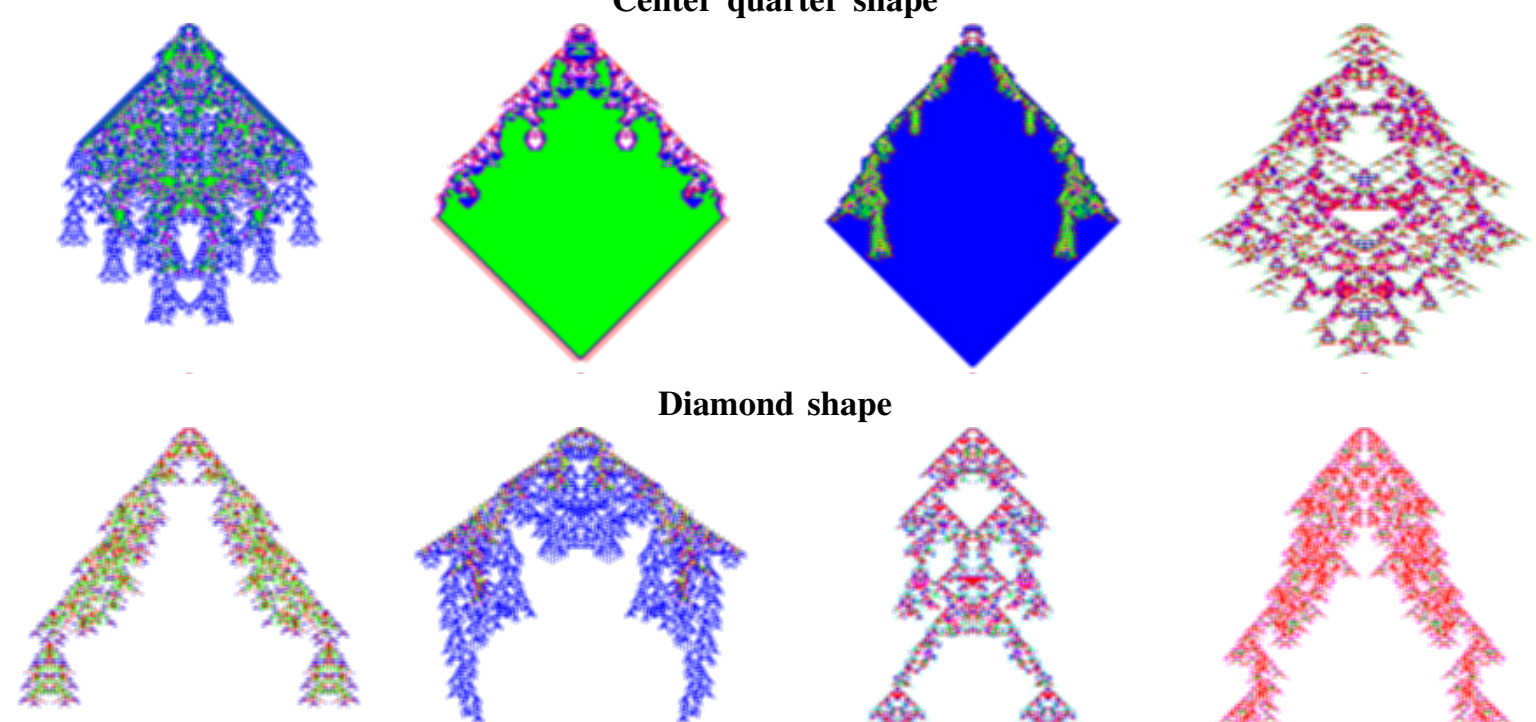

Diamond shape
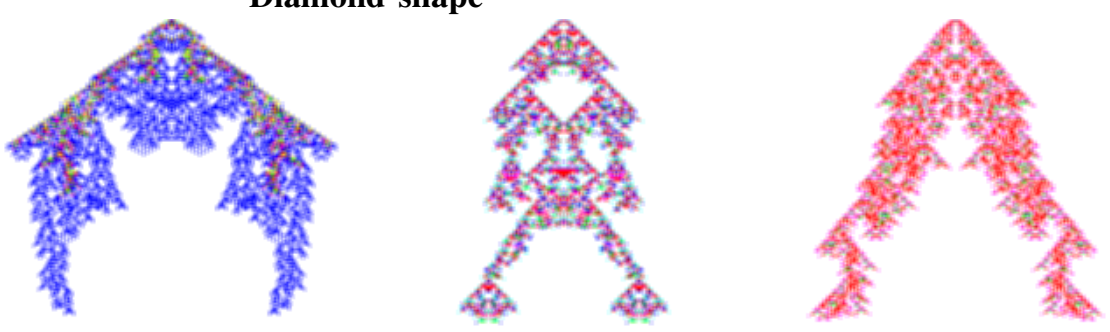

\section{Limited wedge shape}

Fig. 7. Shown are representative renderings from each of the eight experiments with different shapes. The left two columns use the softer selection in which $K=2000$ while the right hand pair of columns were evolved with $K=1000$.

possible applications of necrotic filtering are an early priority for future research.

\section{REFERENCES}

[1] A. Adamatzky, J. Serquera, and E.R. Miranda. Automata-2008: Theory and Applications of Cellular Automata: "Cellular automata sound synthesis: From histograms to spectrograms”. Luniver Press, 2008.

[2] P. Anghelescu. Encryption algorithm using programmable cellular automata. IEEE 2011 World Congress on Internet Security (WorldCIS), pages $233-239,2011$.

[3] D. Ashlock and S. McNicholas. Fitness landscapes of evolved cellular automata. IEEE Transaction on Evolutionary Computation, 15(2):198212, 2013.
[4] D. Ashlock and C. Pugh. Evolutionary cellular automata bonsai. In Proceedings of the 2013 Congress on Evolutionary Computation, Piscataway, NJ, 2013. IEEE Press.

[5] D. Ashlock and J. Tsang. Evolved art via control of cellular automata. In IEEE Congress on Evolutionary Computation, 2009, pages 3338 3344, May 2009.

[6] P. Bentley, J. Greensmith, and S. Ujjin. Two ways to grow tissue for artificial immune systems. In Proc. of the 4th International Conference on Artificial Immune Systems (ICARIS), LNCS 3627, pages 139-152. Springer-Verlag, 2005.

[7] A.A. Burbelko, E. Fras, W. Kapturkiewicz, and D. Gurgul. Modelling of dendritic growth during unidirectional solidification by the method of cellular automata. Materials Science Forum, 649:217-222, 2010.

[8] A.A. Burbelko and D. Gurgul. Simulation of austenite and graphite growth in ductile iron by means of cellular automata. Archives of Metallurgy and Materials, 55(1):53-60, 2010. 


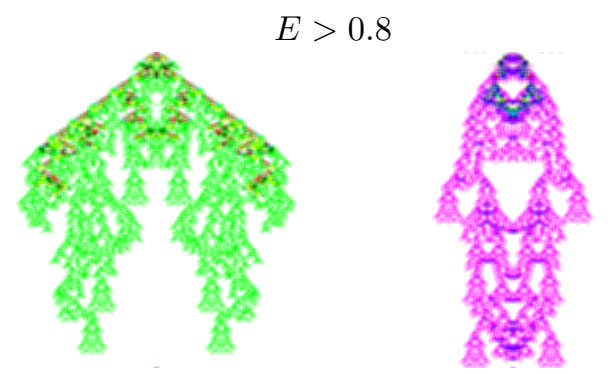

$E>1.0$
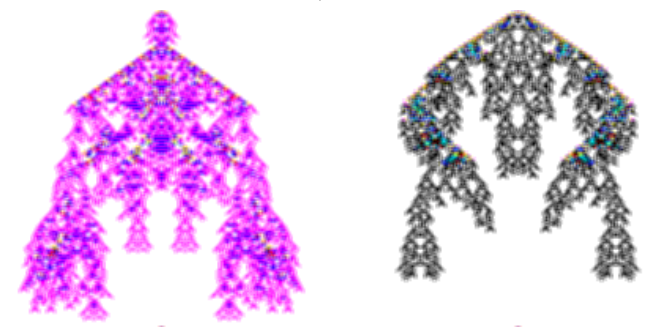

$E>1.2$
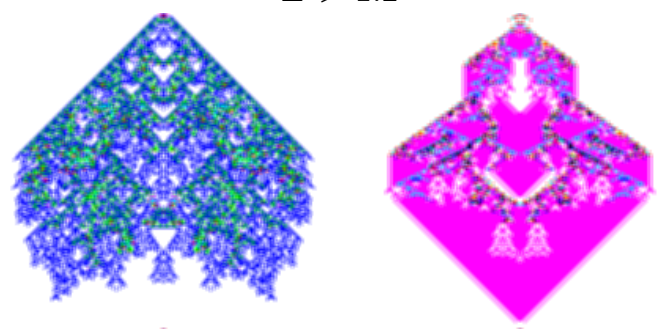

Low Entropy

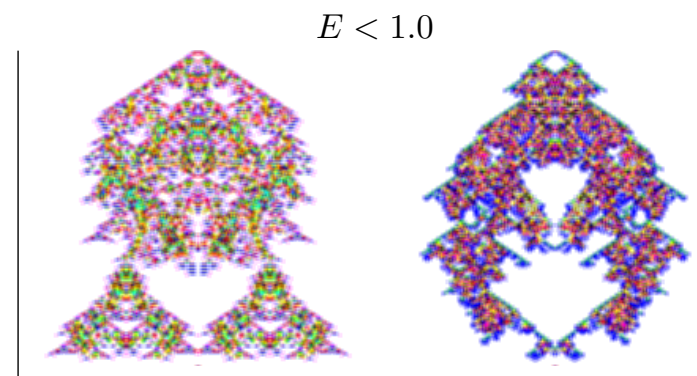

$E<1.2$

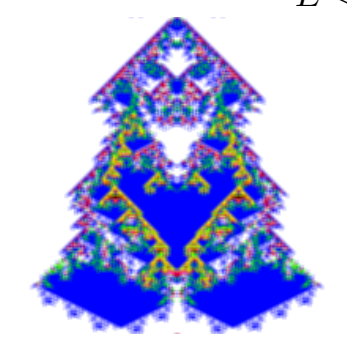

$E<1.4$

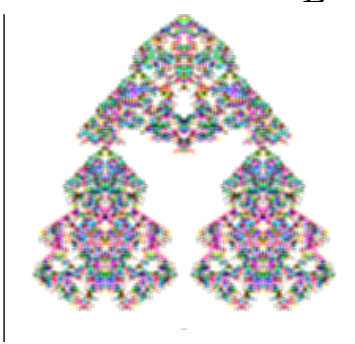

High Entropy
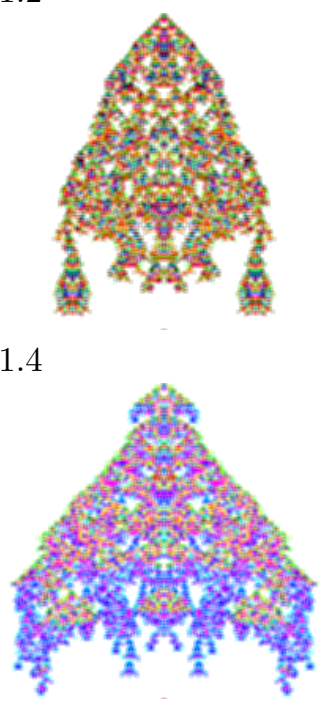$$
\text { , }
$$

sect

Fig. 8. Shown are representative renderings from each of the six experiments with crisp entropic fitness. The left two select for low entropy, the right two select for high entropy. The labels show the definition of what triggers danger for necrosis.

[9] S. Cayzer and U. Aickelin. The danger theory and its application to artificial immune systems. In Proc. of the 1st International Conference on Artificial Immune Systems (ICARIS), pages 141-148. University of Kent at Canterbury Printing Unit, 2002.

[10] Z. Chelly and Z. Elouedi. A survey of the Dendritic Cell Algorithm. Knowledge and Information Systems, 48(3):505-535, 2016.

[11] M. Devetakovic, L. Petrusevski, M. Dabic, and B. Mitrovic. Les folies cellulaires: An exploration in architectural design using cellular automata. 12th Generative Art Conference, pages 181-192, 2009.

[12] N. Elisa, L. Yang, and N. Naik. Dendritic cell algorithm with optimised parameters using genetic algorithm. In 2018 IEEE Congress on Evolutionary Computation (CEC), pages 1-8, 2018.

[13] J. Garner and D. Ashlock. Evolution of 2D apoptotic cellular automata. In Proceedings of the 2015 Congress on Evolutionary Computation, pages 2160-2167, Piscataway, NJ, 2015. IEEE Press.

[14] J. Greensmith, U. Aickelin, and S. Cayzer. Introducing Dendritic Cells as a novel immune-inspired algorithm for anomaly detection. In Proc. of the 4th International Conference on Artificial Immune Systems (ICARIS), LNCS 3627, pages 153-167. Springer-Verlag, 2005.

[15] J. Greensmith, A. Whitbrook, and U. Aickelin. Artificial Immune Systems. In Handbook of Metaheuristics, pages 421-448. Springer, 2010.

[16] Julie Greensmith. Migration threshold tuning in the deterministic dendritic cell algorithm. In International Conference on Theory and Practice of Natural Computing, pages 122-133. Springer, 2019.

[17] M. E. Laragga and L. Alvarez-Icaza. Cellular automaton model for traffic flow based on safe driving policies and human reactions. Physica A, 389(23):5425-5438, 2010.

[18] P. Matzinger. Tolerance, danger and the extended family. Annual Reviews in Immunology, 12:991-1045, 1994.

[19] G. Monro. Emergence and generative art. Leonardo - MIT Press, 42(5):476-477, 2009.
[20] K. Nakamura and K. Imada. Incremental learning of cellular automata for parallel recognition of formal languages. In Proceedings of the 13th international conference on Discovery science, DS'10, pages 117-131, Berlin, Heidelberg, 2010. Springer-Verlag.

[21] T. Pradeu and E.L. Cooper. The danger theory: 20 years later. Frontiers in immunology, 3(287), 2012.

[22] E. Sapin, O. Bailleux, and J. Chabrier. Research of complexity in cellular automata through evolutionary algorithms. Complex Systems, $11,1997$.

[23] S. Sarafijanovic and J. Le Boudec. An artificial immune system for misbehavior detection in mobile ad-hoc networks with virtual thymus, clustering, danger signal and memory detectors. In Proc. of the 3rd International Conference on Artificial Immune Systems (ICARIS), LNCS 3239, pages 342-356, 2004.

[24] J. Serquera and E. R. Miranda. Cellular automata sound synthesis with an extended version of the multitype voter model. In Audio Engineering Society Convention 128, 52010.

[25] J. Serquera and E.R. Miranda. Applications of Evolutionary Computation: "Evolutionary Sound Synthesis: Rendering Spectrograms from Cellular Automata Histograms”. Springer Berlin / Heidelberg, 2010.

[26] V. Singh and N. Gu. Towards an integrated generative design framework. Design Studies, in press, 2011.

[27] H. Situngkir. Exploring ancient architectural designs with cellular automata. BFI Working Paper No. WP-9-2010, 2010.

[28] J. Twycross. Integrated Innate and Adaptive Artificial Immune Systems Applied to Process Anomaly Detection. PhD thesis, University Of Nottingham, 2007.

[29] S. Wolfram. Universality and complexity in cellular automata. Physica D: Nonlinear Phenomena, 10(1-2):1-35, 1984. 